\title{
Inter-sector Interference Mitigation Method in Triple-Sectored OFDMA Systems
}

\author{
JungRyun Lee, Keunyoung Kim, and YongHoon Lim \\ R\&D Center, LG-Nortel Co., Anyang, South Korea \\ \{jylee11, kykim12, yhlim0\}@lg-nortel.com
}

\begin{abstract}
In this paper, a network-centric subcarrier allocation method is proposed for triple-sectored Orthogonal Frequency Division Multiple Access (OFDMA) systems. The proposed method is based on a networkcentric algorithm that is located in a base station (BS) and coordinates the subchannel allocation of each sector. This method controls the resources (subchannels) allocated to each sector, not to waste available bandwidth and avoid inter-sector interference as much as possible. Two methods are suggested for the subchannel allocation method and the detailed algorithms for each method are provided. Simulation results show that the suggested method achieves better throughput than the distributed subchannel and adjacent subchannel configuration methods under a proportional fair algorithm.
\end{abstract}

\section{Introduction}

There has recently been a surge of interest in OFDMA for broadband, high datarate wireless communication. Achieving high transmission rates depends on the system's providing efficient and flexible resource allocation. Recent studies [1[4] on resource allocation demonstrate that significant performance gains can be obtained if scheduling techniques, such as frequency hopping and adaptive modulation, are used in subcarrier allocation, assuming knowledge of the channel gain in the transmitter.

At their most basic, each cell of a cellular system has three sectors, each with its own configuration. To maximize the spectral efficiency in OFDMA system with a triple-sectored cell structure, it is desirable to use an aggressive frequency reuse plan, e.g., the same and whole spectrum is used for multiple neighboring cells and each sector of a cell [5]. In this case, significant cochannel interference is present for sectored OFDMA systems, which results in severe performance degradation, especially at the cell boundaries. To mitigate the inter-cell/sector interference, many interference-tolerant methods have been proposed, such as the Adjacent Permutation Method (APM) and the Distributed Permutation Method (DPM). APM uses adjacent subcarriers to form subchannels. Due to the adjacency of subcarriers, it works well with Adaptive Modulation and Coding (AMC) and Adaptive Array Systems (AAS). DPM allows full-channel diversity by evenly distributing the subcarriers to subchannels.

When APM is used, a subchannel is composed of a branch of adjacent subcarriers and these subcarriers are the same across the cells/sectors if the index 
of the subchannel is the same. In this case, if two or more different cells/sectors allocate the same subchannel at the same time, the throughput performance of the system degrades due to inter-cell/sector interference. In the case of DPM, the number of subcarriers assigned across cells or sectors are proportional to the traffic load 1 of neighboring cells or sectors since the subcarriers are evenly distributed across the subchannels. Thus, average interference in a cell increases as the traffic load of other cells/sectors increases.

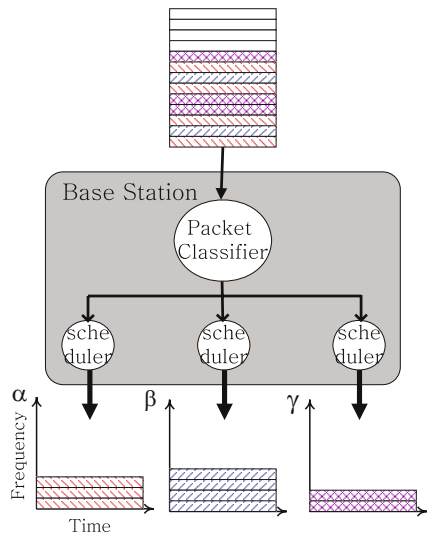

a) blind scheduler

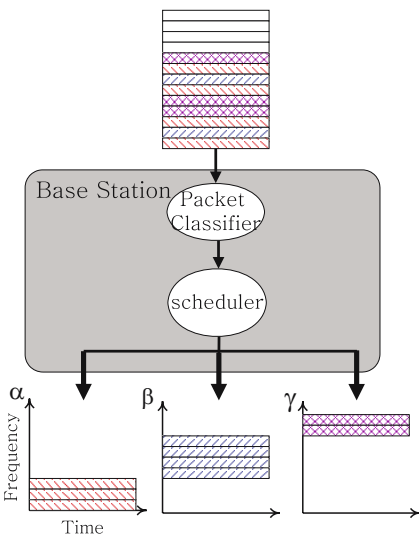

b) BS-centric scheduler

Fig. 1. Blind and BS-centric scheduler concept

The scheduler in each cell or sector may be termed 'blind' in the sense that it does not know which subchannel is allocated in other cells/sectors. In the case of APM, this property may cause significant interference as the number of assigned subchannels across the cells/sectors increases. Fig. 11 a) shows the worst case with respect to intersector interference. Fig. 1 b) shows the ideal subchannel allocation with respect to the reduction of intersector interference. This figure illustrates the necessity of a network-centric method for allocating subchannels. It should be noticed that the proposed network-centric scheduler can be applied across neighboring cells, but, due to the bandwidth limitation of real-time signaling overhead in a multicell environment, we focus on the subchannel allocation strategy in one BS, as shown in Fig. 1.

\section{CINR Calculation}

By intelligently allocating subchannels to each user, we can improve the carrier to interference and noise ratio (CINR). As a result, we can also expect improvement in throughput. The CINR value will differ with different multiple access

${ }^{1}$ The traffic load of each sector is defined as the ratio of the number of subchannels allocated to users to the number of whole subchannels in a sector or cell. 
schemes or with different subchannel permutation methods. We propose a CINR calculation model for each permutation method in OFDMA systems.

It is assumed that power is assigned equally to the subchannels in use and the channel gain of each subchannel is represented by an average value of each subcarrier's channel gain in a subchannel. The interference power in a subchannel from other cells/sectors will be linearly proportional to the number of subcarrier hits 2 in a subchannel. Then, for a receiver belonging to sector $j$, the received power ${ }^{3}$ in $m$-th subchannel from transmitter $i, P_{i, j}^{m}$, is given by

$$
P_{i, j}^{m}=\frac{S_{i}}{N_{i}^{u s e d}} g_{i, j}^{m} \frac{1}{N^{s u b c a}} \cdot \delta_{i, j}^{m},
$$

where $S_{i}$ is the transmitting power from transmitter $i, N_{i}^{\text {used }}$ is the number of allocated subchannels in transmitter $i, g_{i, j}^{m}$ is the average channel gain of $m$-th subchannel between transmitter $i$ and receiver $j, N^{\text {subca }}$ is the number of subcarriers in each subchannel, and $\delta_{i, j}^{m}$ is the number of subcarrier hits in $m$-th subchannel. In eqn (11), $\frac{S_{i}}{N_{i}^{u s e d}} g_{i, j}^{m} \frac{1}{N^{s u b c a}}$ is the received power of each subcarrier. The value of $\delta_{i, j}^{m}$ varies depending on the permutation methods. In DPM, it is assumed that each subchannel in a cell is composed of evenly distributed subcarriers. Thus, the number of subcarrier hits in each subchannel is the same for each subchannel, and $\delta_{i, j}^{m}$ is given by

$$
\delta_{i, j}^{m}=\frac{N^{s u b c a}}{N^{s u b c h}} \cdot N_{j}^{u s e d},
$$

where $N^{\text {subch }}$ is the number of subchannels in each sector. In APM, $\delta_{i, j}$ is given by

$$
\begin{cases}\delta_{i, j}^{m}=N^{\text {subca }}, & \text { if } m \text {-th subchannel is allocated across the sectors } \\ \delta_{i, j}^{m}=0, & \text { otherwise. }\end{cases}
$$

For $i=j$ which is the case that the receiver belongs to the same sector of the transmitter, it is natural that $\delta_{i, j}^{m}=N^{s u b c a}$. Finally, the CINR of $m$-th subchannel for the receiver $i, \gamma_{i}^{m}$ is given by

$$
\gamma_{i}^{m}=\frac{P_{i, i}^{m}}{\sum_{j \neq i} P_{j, i}^{m}+G_{i}}
$$

where $G_{i}$ is additive white Gaussian noise at the receiver $i$.

\footnotetext{
${ }^{2}$ The case that the same subcarrier is assigned across sectors at the same time.

${ }^{3}$ It is assumed that if $i \neq j$, the receiver is located in a different sector of the transmitter, thus, the received power acts as interference power, and if $i=j$, the receiver is located in the same sector of the transmitter, thus, the received power acts as signal power.
} 


\section{Proposed Algorithm}

\subsection{Subchannel Allocation Strategies}

The subchannels are categorized into four classes according to the usage pattern in a triple-sectored cell.

- Class A: subchannel not assigned by any sector.

- Class B: subchannel assigned by only one sector.

- Class $C$ : subchannel assigned by any two sectors.

- Class D: subchannel assigned by all three sectors.

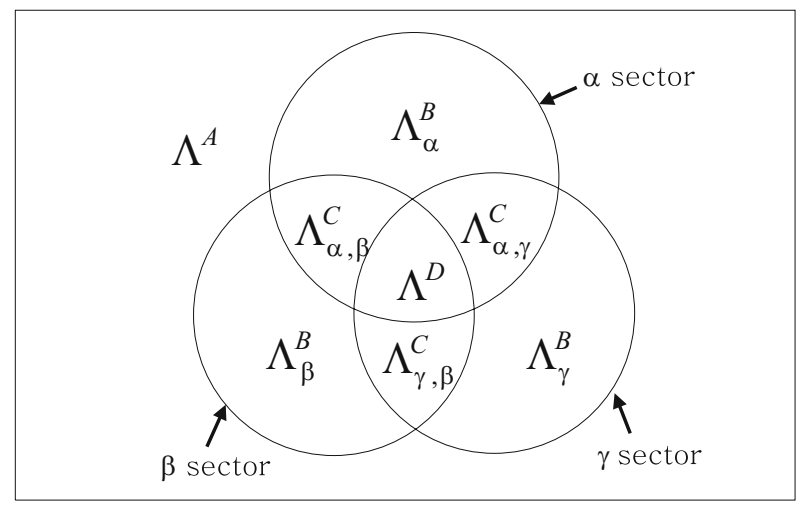

Fig. 2. Schematic representations of used parameters

Let the $\Lambda^{A}, \Lambda^{D}$ be the ratio of subchannels included in Class $A$ and Class $D$ to all available subchannels, respectively. The ratio of subchannels included in sector $i$ and the ratio of subchannels included in both sectors $j$ and $k$ to all available subchannels are defined as $\Lambda_{i}^{B}$ and $\Lambda_{j, k}^{C}$, respectively. Clearly, $\Lambda_{j, k}^{C}=$ $\Lambda_{k, j}^{C}$. The ratio of subchannels included in Class $B$ and Class $C$ to all available subchannels is expressed as $\Lambda^{B}:=\Lambda_{\alpha}^{B}+\Lambda_{\beta}^{B}+\Lambda_{\gamma}^{B}$ and $\Lambda^{C}:=\Lambda_{\alpha, \beta}^{C}+\Lambda_{\beta, \gamma}^{C}+\Lambda_{\gamma, \alpha}^{C}$, respectively. From the above definitions, the following equality is easily verified. (Refer to Fig. 2, )

$$
\Lambda^{A}+\Lambda^{B}+\Lambda^{C}+\Lambda^{D}=1 .
$$

Let the $\omega_{\alpha}, \omega_{\beta}$ and $\omega_{\gamma}$ be the traffic load of $\alpha, \beta$ and $\gamma$ sector, respectively. Our first strategy is to use as many subchannels as possible in a cell (not sectors) to reduce the number of subchannels assigned across sectors. This strategy entails the minimization of $\Lambda^{A}$ (Min Class $A$ ). When $\omega_{\alpha}+\omega_{\beta}+\omega_{\gamma} \leq 1$, this strategy is easily implemented by setting $\Lambda_{\alpha}^{B}=\omega_{\alpha}, \Lambda_{\beta}^{B}=\omega_{\beta}$ and $\Lambda_{\gamma}^{B}=\omega_{\gamma}$ as shown in Fig. 1. b). In this case, $\Lambda^{C}=\Lambda^{D}=0$ and $\Lambda_{A}=1-\left(\omega_{\alpha}+\omega_{\beta}+\omega_{\gamma}\right)$ which is the minimum value. Notice that $\delta_{i, j}=0$ for all subchannels assigned to each 
sector, which means that there is no intersector interference. On the other hand, when $\omega_{\alpha}+\omega_{\beta}+\omega_{\gamma}>1$, the minimum value of $\Lambda^{A}$ is 0 and there should be some subchannels included in Class $C$ or Class D under the Min Class A strategy. To reduce intersector interference, it is desirable to minimize the value of $\Lambda^{C}$ and $\Lambda^{D}$. Specifically, it is more desirable to reduce the value of $\Lambda^{D}$ than that of $\Lambda^{C}$, since a Class $D$ subchannel causes more severe intersector interference than a Class $C$ subchannel. Proposition 1 shows the relationship between the value of Class $C$ and that of Class D.

Proposition 1. Under the assumption that Min Class $A$ is satisfied and the sum of traffic load of each sector is larger than 1, the number of Class C subchannels is minimized (maximized) if and only if the number of Class D subchannels is maximized (minimized).

Proof. From the definitions of used parameters, we derive the following three equations:

$$
\omega_{i}=\Lambda_{i}^{B}+\Lambda_{i, j}^{C}+\Lambda_{i, k}^{C}+\Lambda^{D} \text { for }\{i, j, k\} \in\{\alpha, \beta, \gamma\}
$$

Summing up all equations results in the following equation.

$$
\omega_{\alpha}+\omega_{\beta}+\omega_{\gamma}=\Lambda^{B}+2 \Lambda^{C}+3 \Lambda^{D} .
$$

Eliminating the variable $\Lambda^{B}$ in (7) by applying (5) and $\Lambda^{A}=0$ results in the following equation.

$$
\Lambda^{C}+2 \Lambda^{D}=\omega_{\alpha}+\omega_{\beta}+\omega_{\gamma}-1 \text {. }
$$

Notice that the right side of equation is a fixed value that leads to the conclusion.

From Proposition 1, we can verify that it is not possible to reduce simultaneously all subchannels that induce intersector interference (Class $C$ and Class D subchannels). There are, therefore, two options: to maximize $\Lambda^{C}$ or to maximize $\Lambda^{D}$. Proposition 2 shows the relationship between $\Lambda^{B}$ and $\Lambda^{C}\left(\Lambda^{D}\right)$.

Proposition 2. Under the same assumption as Proposition 1, the number of Class B subchannels is maximized (minimized) if and only if the number of Class $C$ (Class D) subchannels is maximized.

Proof. Eliminating the variable $\Lambda^{C}$ in (7) by applying (5) and $\Lambda^{A}=0$ yields the following equation:

$$
\Lambda^{B}=2-\left(\omega_{\alpha}+\omega_{\beta}+\omega_{\gamma}\right)+\Lambda^{D} .
$$

which leads to the conclusion combining with Proposition 1.

In the Max Class $C$ method, the less likely it is that users will be free from intersector interference (because there are fewer Class $B$ subchannels) or experience severe intersector interference (because there are fewer Class $D$ subchannels). 
As a trade-off, the more likely it is that users will experience less intersector interference than users who are allocated Class $D$ subchannels (because there are more Class $C$ subchannels). By contrast, the Max Class $D$ method ensures the greatest possible number of Class $B$ subchannels. Since Class $B$ subchannels are free from intersector interference, the number of subchannels causing intersector interference is reduced. As a trade-off, the users to whom Class D subchannels are allocated experience more severe intersector interference. The following two subsections describe how to determine the value of $\Lambda_{x}^{Y}$ for Max Class $C$ and Max Class D, respectively. As discussed in the paragraph following Eqn. (6), when $\omega_{\alpha}+\omega_{\beta}+\omega_{\gamma} \leq 1$, it is trivial to meet the Min Class $A$ strategy. Since there is no Class $C$ or Class D subchannel, the Max Class $C$ and Max Class D strategies are meaningless. Thus, the following two subsections consider only the case of $\omega_{\alpha}+\omega_{\beta}+\omega_{\gamma}>1$, thus, $\Lambda^{A}=0$ and the subchannel usage ratid 4 becomes 1 .

\subsection{Subchannel Assignment Method of Max Class C}

Consider the case of $1<\omega_{\alpha}+\omega_{\beta}+\omega_{\gamma} \leq 2$. Suppose all of the subchannels are included in Class B. Then, the subchannel usage ratio becomes $\omega_{\alpha}+\omega_{\beta}+\omega_{\gamma}$, which is larger than 1 . This means that there should be some Class $C$ or Class $D$ subchannels. However, in Max Class $C$, there is no need for Class D subchannels to exist since the subchannel usage ratio becomes $\frac{\omega_{\alpha}+\omega_{\beta}+\omega_{\gamma}}{2}<1$ with the assumption that all subchannels are included in Class $C$. Thus, in this case, all of the subchannels of each sector are included in Class $B$ or Class $C$, and $\Lambda^{D}=0$. The number of subchannels included in Class $C$ in the cell is expressed as

$$
\Lambda^{C}=\frac{\omega_{\alpha}-\Lambda_{\alpha}^{B}+\omega_{\beta}-\Lambda_{\beta}^{B}+\omega_{\gamma}-\Lambda_{\gamma}^{B}}{2} .
$$

So, combining $\Lambda^{A}=\Lambda^{D}=0$ with (5),

$$
\Lambda_{\alpha}^{B}+\Lambda_{\beta}^{B}+\Lambda_{\gamma}^{B}+\frac{\omega_{\alpha}-\Lambda_{\alpha}^{B}+\omega_{\beta}-\Lambda_{\beta}^{B}+\omega_{\gamma}-\Lambda_{\gamma}^{B}}{2}=1 .
$$

We set the proportions among $\Lambda_{\alpha}^{B}, \Lambda_{\beta}^{B}$ and $\Lambda_{\gamma}^{B}$ to $\omega_{\alpha}: \omega_{\beta}: \omega_{\gamma}$ so that the proportion of Class $B$ subchannels in each sector is the same as the proportion of the traffic load in each sector $\left(a_{\alpha}: a_{\beta}: a_{\gamma}=\omega_{\alpha}: \omega_{\beta}: \omega_{\gamma}\right)$. Then, $\Lambda_{\alpha}^{B}$ is given by

$$
\Lambda_{\alpha}^{B}=\frac{\omega_{\alpha}\left(2-\left(\omega_{\alpha}+\omega_{\beta}+\omega_{\gamma}\right)\right)}{\omega_{\alpha}+\omega_{\beta}+\omega_{\gamma}} .
$$

$\Lambda_{\beta}^{B}$ and $\Lambda_{\gamma}^{B}$ are derived in a similar way. On the other hand, $\omega_{\alpha}-\Lambda_{\alpha}^{B}$ subchannels should be included in Class $C$ subchannels. Here, we need to determine the

\footnotetext{
${ }^{4}$ The subchannel usage ratio is defined as the number of subchannels that are allocated to at least one sector divided by the number of subchannels in a cell, namely, $\Lambda^{B}+$ $\Lambda^{C}+\Lambda^{D}$.
} 


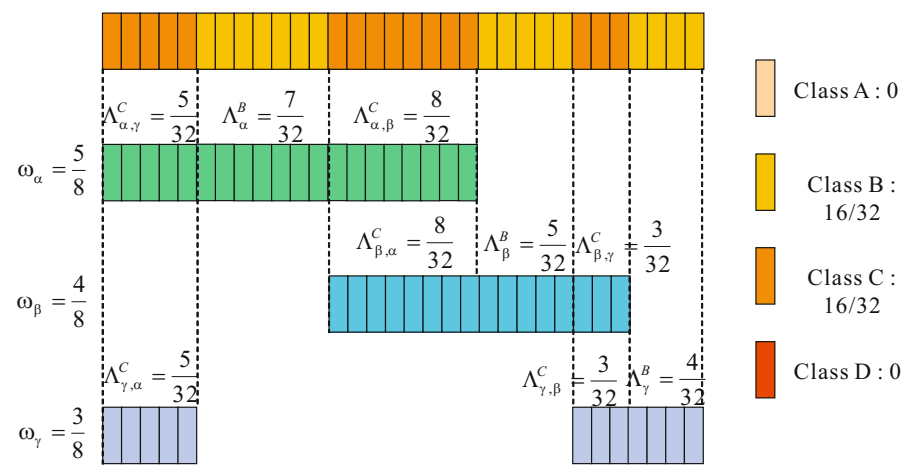

Fig. 3. Example of Max Class $C$ method $\left(\omega_{\alpha}=5 / 8, \omega_{\alpha}=4 / 8\right.$, and $\omega_{\gamma}=3 / 8$.)

exact values of $\Lambda_{\alpha, \beta}^{C}$ and $\Lambda_{\alpha, \gamma}^{C}$. From the following equations (13) and (14), we can obtain the exact value of $\Lambda_{\alpha, \beta}^{C}$ and $\Lambda_{\alpha, \gamma}^{C}$.

$$
\begin{array}{r}
\Lambda_{\alpha, \gamma}^{C}+\Lambda_{\alpha}^{B}+\Lambda_{\alpha, \beta}^{C}=\omega_{\alpha} . \\
\omega_{\alpha}+\left(\omega_{\beta}-\Lambda_{\alpha, \beta}^{C}\right)+\Lambda_{\gamma}^{B}=1 .
\end{array}
$$

The value of $\Lambda_{\beta, \gamma}^{C}$ is derived in a similar way.

Consider the case of $2<\omega_{\alpha}+\omega_{\beta}+\omega_{\gamma} \leq 3$. If all subchannels belong to Class $B$ or Class $C$, the subchannel usage ratio should be larger than 1 , which is absurd. So, there should be Class $D$ subchannels. Since $\omega_{\alpha}+\omega_{\beta}+\omega_{\gamma}>2$, the number of Class $B$ subchannels should be 0 in order to maximize the number of Class $C$ subchannels. Thus, we can get the exact value of $\Lambda^{D}$ from the following equations:

$$
\frac{\omega_{\alpha}-\Lambda^{D}+\omega_{\beta}-\Lambda^{D}+\omega_{\gamma}-\Lambda^{D}}{2}+\Lambda^{D}=1 .
$$

The value of $\Lambda_{i, j}^{C}$ can be obtained based on a derivation procedure similar to that used when $1 \leq \omega_{\alpha}+\omega_{\beta}+\omega_{\gamma}<2$. Fig. 3 shows an example of the Max Class $C$ method when the total traffic load is 1.5. It guarantees the minimum possible number of Class $B$ subchannels, and there are 0 Class $D$ subchannels. Notice that the exact placement of each subchannel may differ, according to the scheduling policy.

\subsection{Subchannel Assignment Method of Max Class D}

We assume $\omega_{\alpha} \geq \omega_{\beta} \geq \omega_{\gamma}$ without loss of generality. The maximum value of $\Lambda^{D}$ becomes $\omega_{\gamma}$. By combining $\omega_{\gamma} \geq \Lambda^{D}$ and (18), the following inequality holds:

$$
\omega_{\alpha}+\omega_{\beta}-\omega_{\gamma}-1 \leq \Lambda^{C}
$$

From Prop. 1. $\Lambda^{C}$ should be minimized under Max Class D. Suppose that $\omega_{\alpha}+$ $\omega_{\beta} \leq \omega_{\gamma}+1$. Then, we can set $\Lambda^{C}=0$. In this case, all subchannels of each 


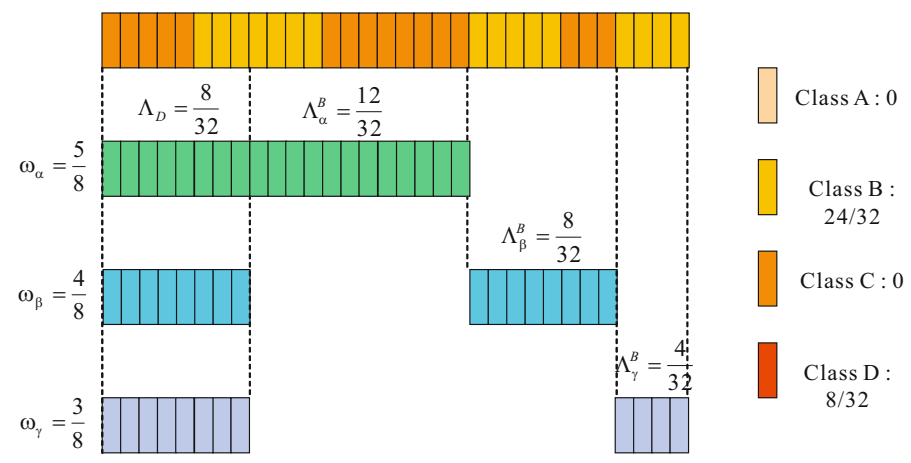

Fig. 4. Example of Max Class D method $\left(\omega_{\alpha}=5 / 8, \omega_{\alpha}=4 / 8\right.$, and $\omega_{\gamma}=3 / 8$.)

sector are included in Class B or Class D. By the Min Class A strategy, we have the following equality:

$$
\left(\omega_{\alpha}-\Lambda^{D}\right)+\left(\omega_{\beta}-\Lambda^{D}\right)+\left(\omega_{\gamma}-\Lambda^{D}\right)+\Lambda^{D}=1 .
$$

From (17), $\Lambda^{D}=\frac{\omega_{\alpha}+\omega_{\beta}+\omega_{\gamma}-1}{2}$, and $\Lambda_{k}^{B}=\omega_{k}-\Lambda^{D}$ follows.

Consider the case of $\omega_{\alpha}+\omega_{\beta}>\omega_{\gamma}+1$. In this case, we set $\Lambda^{D}=\omega_{\gamma}$ which is the maximum value of $\Lambda^{D}$. From (8),

$$
\Lambda^{C}=\omega_{\alpha}+\omega_{\beta}+\omega_{\gamma}-1-2 \Lambda^{D}=\omega_{\alpha}+\omega_{\beta}-\omega_{\gamma}-1 .
$$

From (5), (18) and $\Lambda^{A}=0, \Lambda^{B}=2-\omega_{\alpha}-\omega_{\beta}$. With the same assumption $\left(a_{\alpha}: a_{\beta}: a_{\gamma}=\omega_{\alpha}: \omega_{\beta}: \omega_{\gamma}\right)$ used in subsection $3.2, \Lambda_{\alpha}^{B}$ is given by

$$
\Lambda_{\alpha}^{B}=\frac{\omega_{\alpha}\left(2-\left(\omega_{\alpha}+\omega_{\beta}\right)\right)}{\omega_{\alpha}+\omega_{\beta}+\omega_{\gamma}} .
$$

$\Lambda_{\beta}^{B}$ and $\Lambda_{\gamma}^{B}$ are calculated by similar way. Also, $\Lambda_{i, j}^{C}$ are derived from (13) and (14). Fig. 4 shows an example of the Max Class $D$ method when the total traffic load is 1.5. It guarantees the maximum possible number of Class $D$ subchannels, and there are 0 Class $B$ subchannels since $\omega_{\alpha}+\omega_{\beta} \leq \omega_{\gamma}+1$. As with Max Class $C$, the exact placement of each subchannel may differ according to the scheduling policy.

\section{Simulation Design}

For simulation environment, 1024-FFT for $10 \mathrm{MHz}$ bandwidth is assumed for an OFDMA channel configuration. The number of subchannels $\left(N^{s u b c a}\right)$ for APM and DPM are the same as 32. Simulation runs were based on the Monte Carlo method with 100,000 trials. In each trial, 10 users were generated in the center cell and the locations of users were uniformly distributed in the cell. We assumed that each user always had traffic to be sent, which setup is usually called a full-buffered traffic model. The center cell was surrounded by 18 
Table 1. CINR - data rate mapping table

\begin{tabular}{||c|c||c|c||}
\hline Min CINR[dB] & Data rate[kbps] & Min CINR[dB] & Data rate[kbps] \\
\hline \hline-6.6 & 38.4 & 7.8 & 460.8 \\
\hline-4.1 & 57.6 & 12.3 & 691.2 \\
\hline-1.1 & 115.2 & 15.4 & 921.6 \\
\hline 2 & 230.4 & 18.5 & 1152 \\
\hline
\end{tabular}

cells which contribute intercell interference, and the radius of each cell was assumed to be $1 \mathrm{~km}$. The antenna pattern in a triple-sectored cell is expressed as $A(\theta)=-\min \left[12\left(\frac{\theta}{70}\right), 20\right]$ where $-180 \leq \theta \leq 180$ [6]. We assumed that intercell interference resulted from fully-loaded cell. That is, all subchannels and maximum power are used in other cells. The interference from other sectors in the center cell was calculated from the actually allocated subchannels according to Max Class C, Max Class D, APM and DPM, respectively. The traffic load in the center cell was averaged over $\alpha, \beta$ and $\gamma$ sectors where $\omega_{\alpha}=(i+1) / 8, \omega_{\beta}=i / 8$, and $\omega_{\gamma}=(i-1) / 8$ for $1 \leq i \leq 7$. For the channel model, we considered only a path-loss model [7], which is given by $L=35.2 \log _{10} D+137.32$ where $D$ is the distance between a transmitter and a receiver. For scheduling policy, we used a proportional fair algorithm with window size 1000 that acknowledges feedback about the channel quality for each user. It chooses the user $i$ that maximizes $\frac{D R C_{i}(t)}{R_{i}(t)}$ where $R_{i}(t)$ is an exponentially smoothed average of the service rate received by user $i$ and $D R C_{i}(t)$ is the amount of data that can be transmitted to user $i$ in time slot $t$ [ .

The system performance of the proposed algorithm was evaluated by the average throughput of the target cell. Average throughput was derived from the CINR-throughput relationships which resulted from the link level simulation results combining with AMC operation. Table 1 shows the mapping table between CINR and achievable data rates.

\section{Result}

Fig. 5 shows the average throughput as a function of average traffic load of the target cell. The result shows that average throughput of Max Class $C$ is better than that of APM, DPM and Max Class D. However, when the average traffic load is high, the gain from the proposed algorithms is less, since there is less opportunity for the proposed algorithm to select the subchannels included in Class B, and almost all subchannels belong to Class $C$ or Class D. The difference in throughput gain between Max Class $C$ and Max Class D increases as the offered traffic load increases, which indicates that Max Class $C$ performs better than Max Class D. This result means that, with respect to mitigation of intersector interference, it is desirable to avoid the Max Class D method as traffic load is increasing, even though there are more Class $B$ subchannels under Max Class $D$ method. Furthermore, the throughput of Max Class D is worse than that of APM when the traffic load is high. However, when the average traffic load 


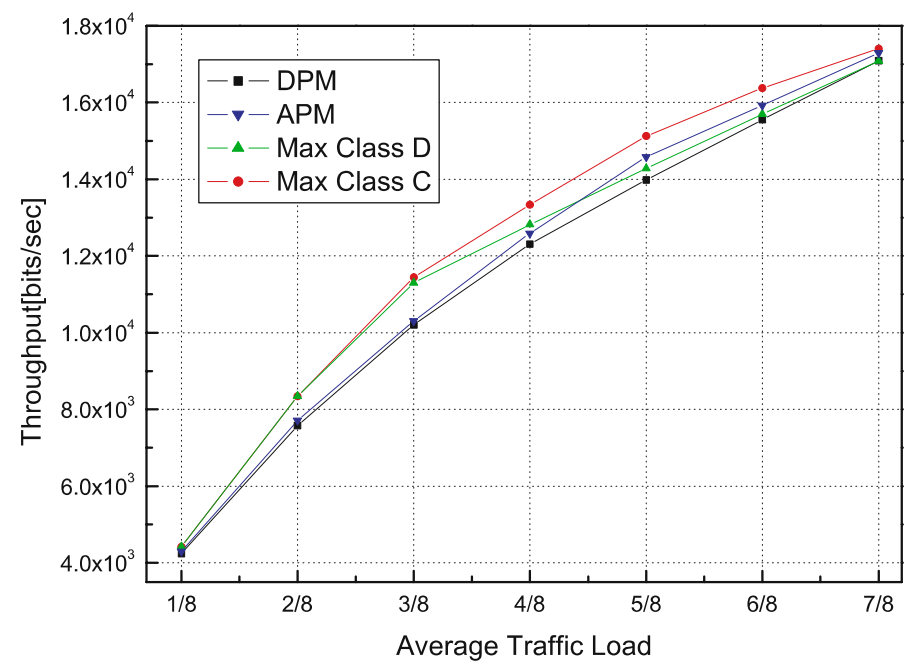

Fig. 5. Throughput as a function of average traffic load

is less then 3/8, the throughput difference between Max Class C and Max Class $D$ is negligible because almost all the subchannels of each sector are expected to be included in Class B. (Notice that when the sum of the traffic loads of each sector is less than 1, all subchannels are included in Class $B$ subchannel by the Min Class A strategy.)

\section{Conclusions}

We proposed a BS-centric method for subchannel allocation, based on APM in a triple-sectored BS. When using this algorithm, signaling cost and required bandwidth are very small, due to the adjacency of each sector. We classified the subchannels into four categories according to the usage pattern in the BS. By minimizing the number of Class A subchannels, the whole spectrum of the base station is divided efficiently in the service of mitigating intersector interference. With the assumption of the Min Class A method, Max Class C and Max Class D methods are proposed and the detailed algorithms to realize the proposed two methods are suggested. Simulation results show that, with respect to average throughput of the target cell, the proposed Max Class $C$ method performs better than APM, FPM and Max Class D under a proportional fair algorithm with window size 1000 .

\section{References}

1. I. Koutsopoulos and L. Tassiulas, "Channel state-adaptive techniques for throughput enhancement in wireless broadband networks", INFOCOM 2001, vol. 2, pp. 757-766, 2001. 
2. C. Y. Wong, R. S. Cheng, K. B. Letaief, and R. D. Murch, "Multiuser OFDM with adaptive subcarrier, bit, and power allocation", IEEE J. Select. Areas Commun., vol. 17, no. 10, pp. 1747-1758, Oct. 1999.

3. Y. Zhang and K. B. Letaief, "Multiuser subcarrier and bit allocation along with adaptive cell selection for OFDM transmission", ICC 2002, vol. 2, pp. 861-865, 2002.

4. M. Ergen, S. Coleri and P. Varaiya, "QoS aware adaptive resource allocation techniques for fair scheduling in OFDMA based broadband wireless access systems", IEEE Trans. on Broadcasting, vol. 49, no. 4, pp. 362-370, Dec. 2003.

5. A. Ghosh et al, "Broadband Wireless Access with WiMax/802.16: Current Performance Benchmarks and Future Potential", Communications Magazine, IEEE, vol. 43, no. 2, pp.129-136, Feb. 2005.

6. 3GPP2 C.R1002-0, "cdma2000 Evaluation Methodology", Wireless Networks, Dec. 2004.

7. ITU-R, M.1225, "GUIDELINES FOR EVALUATION OF RADIO TRANSMISSION TECHNOLOGIES FOR IMT-2000", 1997.

8. D. M. Andrews, "Instability of the proportional fair scheduling algorithm for HDR", IEEE Trans. Wireless Commun., vol. 3, no. 5, pp. 1422-1426, Sep. 2004. 